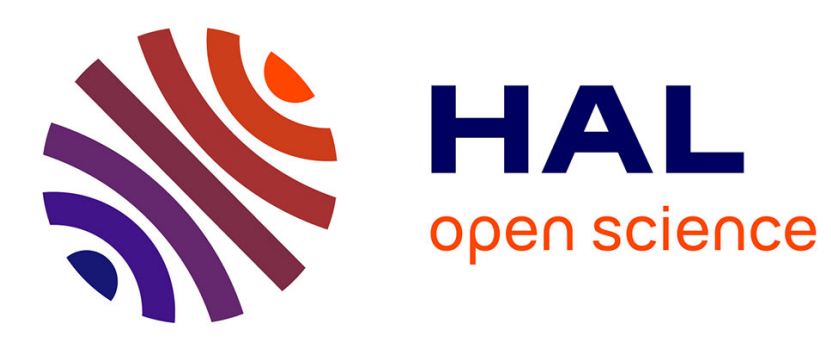

\title{
The Corona Limit of Penrose Tilings Is a Regular Decagon
}

Shigeki Akiyama, Katsunobu Imai

\section{To cite this version:}

Shigeki Akiyama, Katsunobu Imai. The Corona Limit of Penrose Tilings Is a Regular Decagon. 22th International Workshop on Cellular Automata and Discrete Complex Systems (AUTOMATA), Jun 2016, Zurich, Switzerland. pp.35-48, 10.1007/978-3-319-39300-1_4 . hal-01435032

\section{HAL Id: hal-01435032 \\ https://hal.inria.fr/hal-01435032}

Submitted on 13 Jan 2017

HAL is a multi-disciplinary open access archive for the deposit and dissemination of scientific research documents, whether they are published or not. The documents may come from teaching and research institutions in France or abroad, or from public or private research centers.
L'archive ouverte pluridisciplinaire HAL, est destinée au dépôt et à la diffusion de documents scientifiques de niveau recherche, publiés ou non, émanant des établissements d'enseignement et de recherche français ou étrangers, des laboratoires publics ou privés. 


\title{
The corona limit of Penrose tilings is a regular decagon
}

\author{
Shigeki Akiyama ${ }^{1}$ and Katsunobu Imai ${ }^{2}$ \\ 1 Institute of Mathematics \& Center for Integrated Research in Fundamental Science \\ and Technology, University of Tsukuba \\ Tennodai, Tsukuba, Ibaraki, 305-8571 Japan \\ akiyama@math.tsukuba.ac.jp \\ 2 Graduate School of Engineering, Hiroshima University, \\ Higashi-Hiroshima 739-8527 Japan \\ imai@hiroshima-u.ac.jp
}

\begin{abstract}
We define and study the corona limit of a tiling, by investigating the signal propagations on cellular automata (CA) on tilings employing the simple growth CA. In particular, the corona limit of Penrose tilings is the regular decagon.
\end{abstract}

\section{Introduction}

Since the discovery of quasi-crystals, quasi-periodic tilings like Penrose tiling attracted a lot of attention as their possible mathematical models. Spectral study of tiling dynamical system and Schrödinger operator on quasi-periodic structure are developed in order to analyze their long-range order and quantum mechanical motion of the particle on quasi-periodic structure [8].

Cellular automata working on quasi-periodic tilings $[11,2,16]$ are also studied. In particular, the intensive studies of the Game of Life [3] on Penrose tilings by Owens and Stepney are paid attentions $[12,13]$. As the result, recently, a cellular automata simulator for reaction-diffusion media which also working on a Penrose tiling is released [7] and the first glider pattern on Penrose tilings is also found [5].

We are interested in the difference of signal propagation of cellular automata working on between the normal periodic cells and quasi-periodic tilings. In this context, Chidyagwai and Reiter showed that the broken symmetry of quasiperiodic tilings, while still retaining a highly organized structure, could be used to simulate the complex growth of snow crystals [2]. They could produce global $n$-fold symmetry models where regular hexagonal grids could only produce 6 -fold symmetry models.

In this paper, we introduce corona limit which naturally visualizes the growth pattern of signal propagation. We show that the corona limit of a Penrose tilings is a regular decagon. The speed of convergence depends on the version of tilings and adjacency condition of it. 


\section{Corona limit}

A tiling $\mathcal{T}$ is a covering of $\mathbb{R}^{2}$ by finitely many polygonal tiles and their images by isometry (translation, rotation, and flip) which overlap only at their boundaries. Two tiles $A, B$ are adjacent (resp. edge adjacent) if they share a point (resp. an edge). For brevity, we also say that $A$ and $A$ itself are adjacent (edge adjacent) as well. A patch $\mathcal{P}$ is a finite set of tiles in $\mathcal{T}$. The 1 -st corona $\mathcal{P}^{(1)}$ of $\mathcal{P}$ is a patch consisting of all tiles which is adjacent to a tile of $\mathcal{P}$. The $n$-th corona $\mathcal{P}^{(n)}$ is defined as a corona of the $(n-1)$-th corona $\mathcal{P}^{(n-1)}$ for $n=2,3, \ldots$. An edge corona $\mathcal{P}^{[1]}$ and $n$-th edge corona $\mathcal{P}^{[n]}$ are defined in the same manner but by edge adjacency. The concept of corona is important and well-known, which is used in the basic theory of tilings, e.g. [6, Chap 3.2], [15] and [14]. It also appears in Heesch's problem ([6, Chap 3.8.3] and [10]) which forms a counter part of existence problem of tilings by a given set of tiles. If a sequence of shrunk patches

$$
\frac{1}{n} \mathcal{P}^{(n)} \quad n=1,2, \ldots
$$

converges to a non-empty compact set $K$ under Hausdorff metric, we say that $K$ is a corona limit. Here for two non empty compact sets $A$ and $B$, the Hausdorff metric is defined as

$$
\inf \{\varepsilon>0 \mid A \subset B[\varepsilon] \text { and } B \subset A[\varepsilon]\}
$$

with $X[\varepsilon]:=\left\{y \in \mathbb{R}^{2} \mid \exists x \in X\|y-x\| \leq \varepsilon\right\}$. To see the geometric meaning better, the limit is rephrased as $\lim _{n} \frac{1}{n}\left(\mathcal{P}^{(n)}-c\right)$ by fixing a point $c$ in the initial patch $\mathcal{P}$. Then the coronas grow around the center $c$, and we renormalize them by the factor $1 / n$ to obtain the corona limit. Since the tiling has finitely many shapes, the diameter and inradius (maximal radius of the inscribed ball) of the $n$-th corona are bounded from below and above by positive constant multiples of $n$. Thus the corona limit contains the origin as an inner point, and is bounded. Interestingly, one can show that if a corona limit $K$ exists, then it does not depend on the choice of the initial patch $\mathcal{P}$. To see this, we claim that the corona limit of $\mathcal{P}$ and that of $\mathcal{P}^{(n)}$ are identical, and for any two patches $\mathcal{P}, \mathcal{Q}$, there exist a positive integer $m$ that $\mathcal{Q} \subset \mathcal{P}^{(m)}$ and $\mathcal{P} \subset \mathcal{Q}^{(m)}$. Therefore we say that $K$ is the corona limit of $\mathcal{T}$. We can similarly define the edge corona limit. For periodic tilings, the corona limit is usually easy to obtain by simple induction. For the standard square tiling the corona limit is a square, and the edge corona limit is also a square but rotated $\pi / 4$. It often becomes a hexagon for Archimedean tilings.

\section{Penrose Tilings}

A Penrose tiling is a tiling generated by a set of tiles with matching conditions. They tiles the plane but only in non periodic way. Among many versions of Penrose tilings, there are two types of tilings generated by two quadrilateral proto-tiles : two rhombus tiles (a fat and a thin), and a kite and a dart. To avoid 
periodic arrangements, several matching conditions are known to be added on these tiles [4]. Ammann bars are one of such matching condtions [6]. Each tile in Fig. 1 has Ammann bar line segments and Fig. 2 illustrates a possible rhombus tiling and its substitution rules. Let $\varphi$ be the golden ratio. To get a tiling from a patch, iterate the magnification by $\varphi$ and substitution for each tile by the rule in Fig. 2. The line segments of each tile must be continued straight across the boundary. They form parallel lines of five different slopes and the gap length of each parallel lines is one of L and S. It is known that there exist infinitely many translationaly inequivalent quasi-periodic tilings of the plane with these tiles.
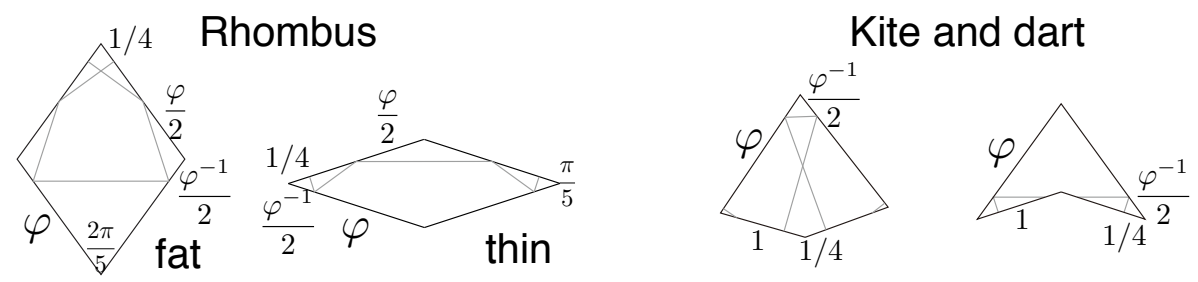

Fig. 1. Rhombus, and kite and dart tiles with the line segments of Ammnann bars.
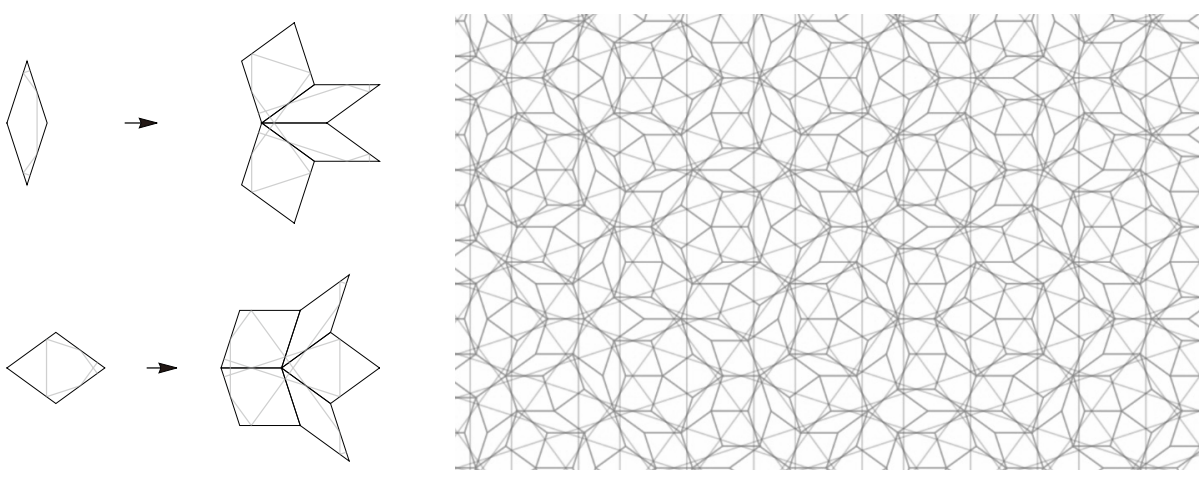

Fig. 2. A rhombus tiling and its substitution rules.

\section{Growth Cellular Automaton on Penrose Tilings}

We define a cellular automaton $\mathcal{A}$ as follows: choose a two-dimensional tiling $\mathcal{T}$. Each tile $\tau$ has a state $c(\tau) \in Q$ and neighborhood tiles are defined by $\mathcal{N}(\tau)$. 
We call all assignments of state to the tiles $\mathcal{C}: \mathcal{T} \rightarrow Q$ as the configuration of $\mathcal{A}$. The local function of $\mathcal{A}$ is defined by the assignment of states in $\mathcal{N}(\tau)$ and returns the next state of $\tau$. Thus its simultaneous application to each tile in $\mathcal{C}$ defines the global evolution $\mathcal{A}: \mathcal{C} \rightarrow \mathcal{C}$. When the tiling is the regular square grid, we denote the vertex adjacent neighborhood by Moore neighborhood $\left(\mathcal{N}_{M}\right)$ and the edge adjacent neighborhood by von Neumann neighborhood $\left(\mathcal{N}_{N}\right)$.

In order to define a $\mathrm{CA}$ on a Penrose tiling, we need to extend the above neighboring relation. Because it is not a lattice tiling, congruent tiles may have different neighborhoods. Generalized von Neumann and Moore neighborhoods are illustrated in Fig. 3 and Fig. 4, respectively [12].

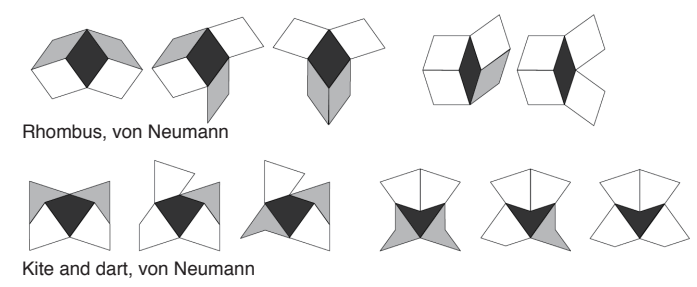

Fig. 3. The generalized von Neumann neighborhoods on Penrose tilings [12]

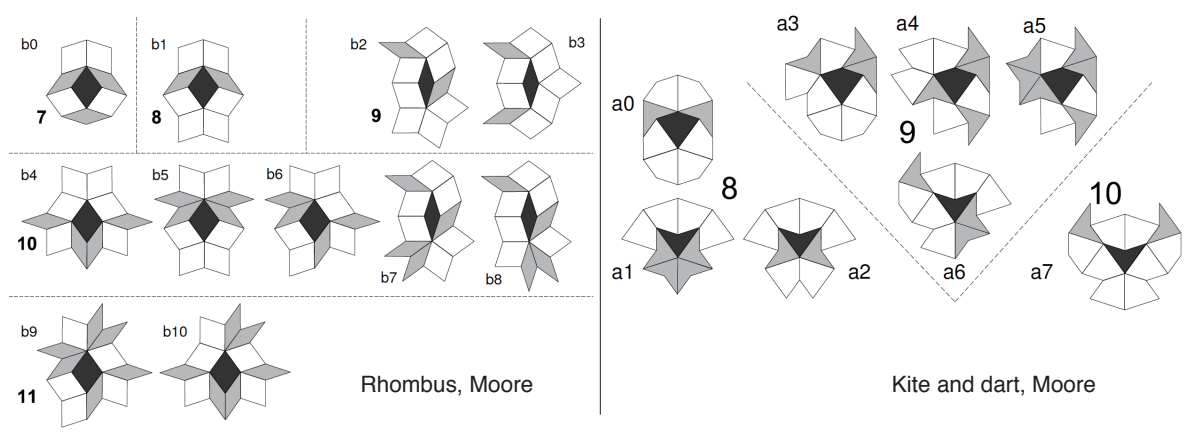

Fig. 4. The generalized Moore neighborhoods on Penrose tilings [12]

From this section, we focus on a simple cellular automaton, growth cellular automaton.

Definition 1. Growth cellular automaton is a cellular automaton $\mathcal{A}$ whose state set is $Q=\{0,1\}$. Its local function is defined such that if the state of a focus tile 
is 0 and at least one of its neighborhood tiles' state is 1 then its state changes to 1. Any tile of state 1 never changes its state.

Let $C_{0}$ be a configuration such that a single cell's state is 1 and the others are 0 . Let $P_{n}$ be the shape formed by state 1 cells by $n$-step evolutions of $\mathcal{A}$ from $C_{0}$ as its initial configuration.

It is clear that in the case of square Moore neighborhood, $P_{n}$ is a square which side length is $2 n+1$ and in the case of square von Neumann neighborhood, $P_{n}$ is a $\pi / 4$-rotated square which diagonal length is $2 n+1$.

We show the simulated results of the case of Penrose tilings. Fig. 5 illustrates the results after 10-step and 30-step evolutions (The scale of the figures of 10 and 20 steps are different). Because the $\left|\mathcal{N}_{M}\right|$ is larger than $\left|\mathcal{N}_{N}\right|$ on average, the size of $P_{n}$ is larger in the case of Moore neighborhoods than the case of von Neumann neighborhood. Each $P_{n}$ seems to converge to a regular decagonal shape. Even the case of rhombus Moore neighborhood, it eventually converges to a regular decagonal shape (See Section 6).

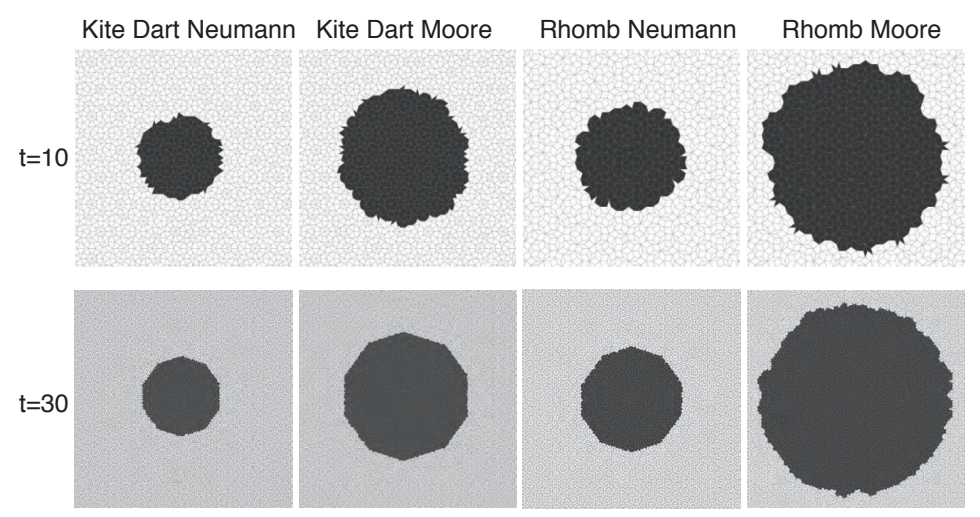

Fig. 5. The evolution of growth CA $\mathcal{A}$ on Penrose tilings ( $t=10,30$ steps).

In the next section we show its proof in the case of rhombus von Neumann neighborhood.

\section{Rhombus von Neumann neighborhood case}

In this section, we prove the experimental observation of the previous section in the case of rhombus von Neumann neighborhood.

Choose a patch of the star shape in any rhombus Penrose tiling $\mathcal{T}$ as in Fig. 6. It has five symmetric crossing Ammann bars. We denote the central point of the star by $O$. We call the five symmetric Ammann bars across the star as $a_{0}, b_{0}, c_{0}, d_{0}, e_{0}$. We denote by $\varepsilon$ the distance between $O$ and one of the 
Ammann bars. We denote by $X_{i}$ for each Ammann bar parallel to $X_{0}$ where $X \in\{a, b, c, d, e\}$ and for all integers $i$. We also denote $X_{-i}$ by $\bar{X}_{i}$. The length of the gap between two Ammann bars $X_{i}$ and $X_{i+1}$ (denoted by $\bar{X}_{i} X_{i+1}$ ) is L or S. We denote by $X_{i} Y_{j}$ the cross point of two non-parallel Ammann bars $X_{i}$ and $Y_{j}$.

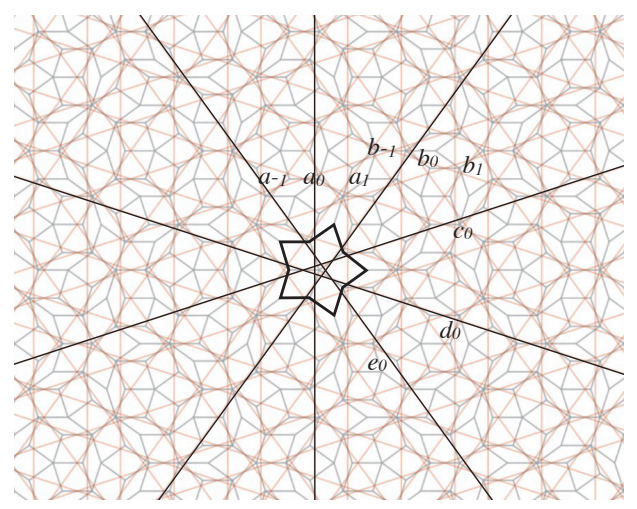

Fig. 6. Amman bars and their indices.

Proposition 1. Let $\partial D_{i}$ be connected lines formed by the points: $a_{i} b_{i}, b_{i} c_{i}, c_{i} d_{i}$, $d_{i} e_{i}, e_{i} \bar{a}_{i}, \bar{a}_{i} \bar{b}_{i}, \bar{b}_{i} \bar{c}_{i}, \bar{c} \bar{d}_{i}, \bar{d} \bar{e}_{i}, \bar{e}_{i} a_{i}, a_{i} b_{i}$. There exists a constant $k$, for any $i(>k)$, $\partial D_{i}$ forms a decagon $\left(D_{i}\right)$ and $D_{i} \subset D_{i+1}$.

Proof. We denote by $\hat{X}_{i}$ the distance between $O$ and $X_{i}$. For any two Ammann bars $X_{i}$ and $Y_{i}$ which angle is $\pi / 5$, if $\hat{X}_{i} / \hat{Y}_{i}>\cos (\pi / 5)$ then $\partial D_{i}$ forms a decagon. Because $\hat{X}_{i}=\overline{X_{0}, X_{i}} \pm \varepsilon$ and $\overline{X_{0}, X_{i}}$ is the $\mathrm{i}$-th addition of $\mathrm{L}$ or $\mathrm{S}$, the value $\hat{X}_{i} / \hat{Y}_{i}$ converges to 1 as $i$ grows. So there exists a constant $k$, for any $i(>k)$, $\hat{X}_{i} / \hat{Y}_{i}>\cos (\pi / 5)$.

We call $D_{i}$ (where $i$ satisfies that each $D_{i}$ forms a decagon) as a sequence of uniaxial Ammann bar decagons (Fig. 7).

Proposition 2. Decagon $D_{i}$ converges to the shape of regular decagon as $i$ goes to infinity.

Proof. Because the ratio of the numbers of L and S for parallel Ammann bars converges to $\varphi$ (10.6.8 in [6], [9]), i.e., for any large $i$, the distance between $O$ and $X_{i}$ converges to the same length. Thus $D_{i}$ converges to a regular decagon as $i$ goes to infinity.

Definition 2. If the part of the area of a tile $\tau$ in the gap between two Ammann bars $X_{i}$ and $X_{i+j}$ is larger than the remaining part, then we call $\tau$ is a gap tile of $X_{i}$ and $X_{i+j}$. We denote by $G\left(X_{i}, X_{i+j}\right)$ all gap tiles of $X_{i}$ and $X_{i+j}$. 


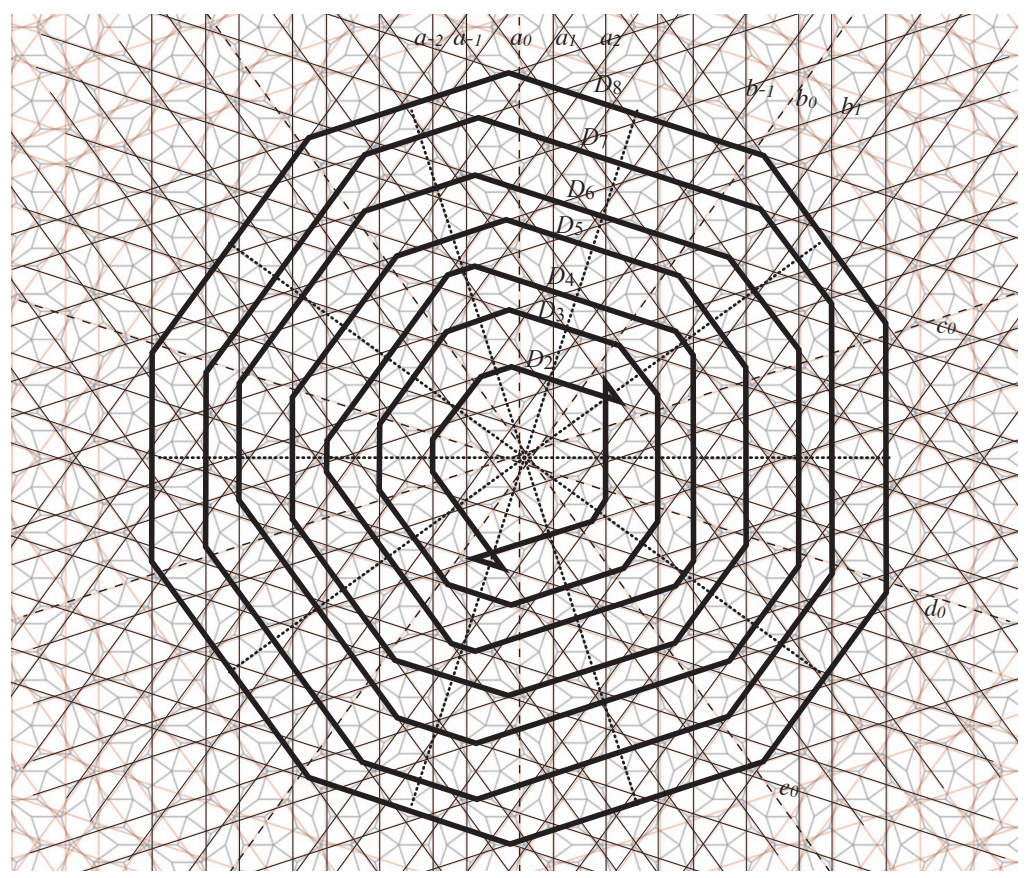

Fig. 7. A sequence of uniaxial Ammann bar decagons $D_{i}(i \geq 3)$

Definition 3. $G\left(X_{i}, X_{i+1}\right)$ is said to be filled when the state of all tiles in $G\left(X_{i}, X_{i+1}\right)$ is 1 except fat tiles of which longer diagonal lines are perpendicular to the bar $X_{i}$ or $X_{i+1}$. We do not concern the states of these fat tiles.

Fig. 8 shows a filled S-gap and a filled L-gap.

Proposition 3. For any tile $\tau \in G\left(X_{i}, X_{i+1}\right)$, the following properties hold:

1. $\tau$ intersects only one of two Ammann bars $X_{i}$ and $X_{i+1}$;

2. If $\tau$ intersects $X_{i}\left(X_{i+1}\right)$ then there exists an edge adjacent tile in $G\left(X_{i}, X_{i+1}\right)$ which intersects the bar $X_{i+1}\left(X_{i}\right)$ and $\tau$ has no adjacent tile in $G\left(X_{i+1}, X_{i+2}\right)\left(G\left(X_{i-1}, X_{i}\right)\right)$;

3. If $\tau$ intersects $X_{i}\left(X_{i+1}\right)$, then $\tau$ has an edge adjacent tile in $G\left(X_{i-1}, X_{i}\right)$ $\left(G\left(X_{i+1}, X_{i+2}\right)\right)$.

Proof. There are 16 possible combinations of two tiles. Removing rotationally symmetric pairs, there are only eight pairs in Fig. 9. Moreover one of them is an illegal combination, i.e., if the pattern is included, some of its Ammann bar segments cannot form lines. The pairs L1 (S1, S2) is the component of two pairs of parallel Ammann bars with L-gaps (S-gaps), respectively. The pairs L2 and L3 are the component of one pair of parallel Ammann bars with L-gaps and they are symmetric with each other. The pairs LS1 and LS2 are the component of parallel Ammann bars with an L-gap and an S-gap and they are also symmetric 


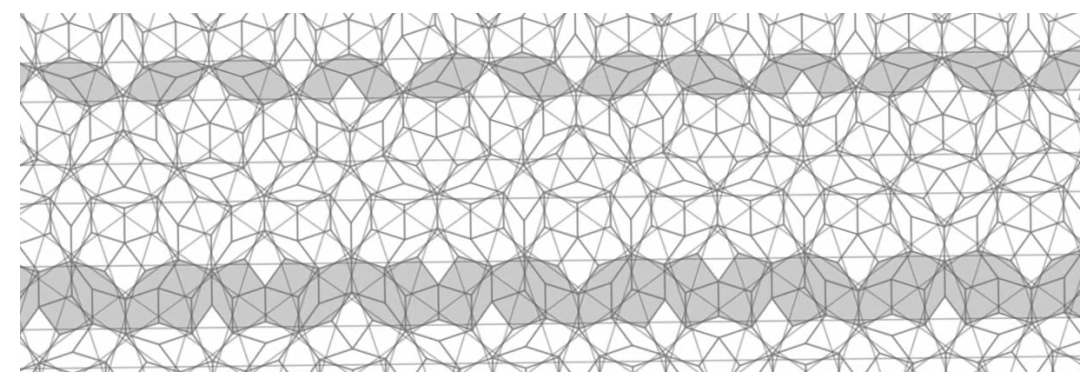

Fig. 8. S- and L-filled gaps.

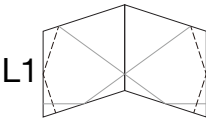

L

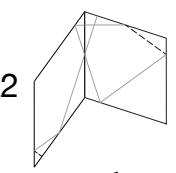

L3

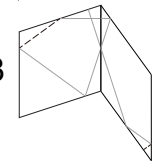

LS1

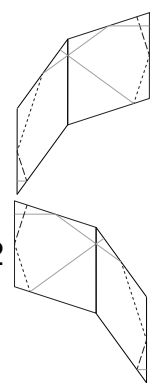

S1
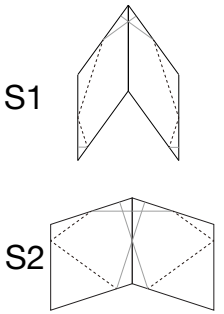

Illegal pair

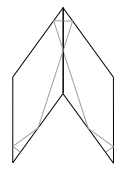

Fig. 9. Possible pairs according to Ammann bars.

with each other. Dashed (dotted) line segments are used for L-gaps (S-gaps), respectively.

To exhaust the possible placements along an S-gap, we start from a pair S1. Although S1 contains line segments for two distinct S-gaps, they are symmetric. So it is enough to consider one pair of them. Fig. 10(1) shows the first possible extensions. The right patch in Fig. 10(1) is the only possibility because the left patch contains some Ammann bar segments which cannot form limes.

The second step, there are three possibilities in Fig. 10(2). S1 appears again in Fig. 10(2)-3. Fig. 10(2)-1 and Fig. 10(2)-2 have a fat tile of the same angle along the S-gap. But the latter is an illegal placement because there is no feasible placement in the next extension. Thus we can continue the next step only in the case of 1 . The final step, the only possible placement is Fig. 10(3) and S1 appears again.

Fig. 11 is the possible extensions to the opposite direction from a pair S1. Because any thin tile cannot be placed to the opposite side of S1 as in Fig. 11(1), fat tiles forced to be placed next to S1. Fig. 11(2)-1 and 2 have already appeared in Fig. 10(2)-1 and 2, respectively. S1 appears again in Fig. 11(2)-3. Thus there is no different placement except their symmetric cases appeared in the both side of S1.

The downside (upperside) of S2 appears in Fig. 10(1) right (Fig. 10(2)), respectively. The upper side of LS1 (and the symmetric version, LS2) appears 
(1) 1st extension

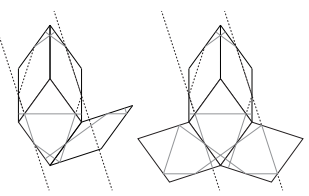

illegal
(2) 2nd extension

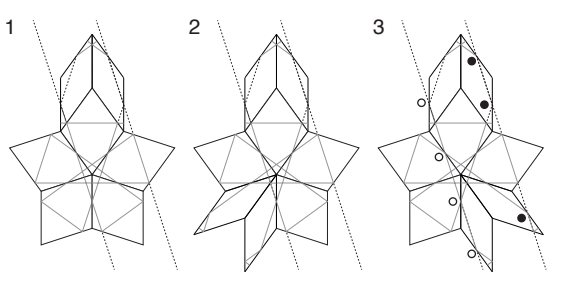

(3) 3rd extension

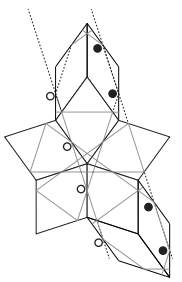

Fig. 10. Possible extensions from a pair S1.

(1) 1st extension

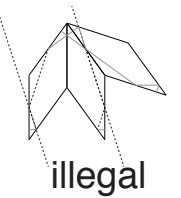

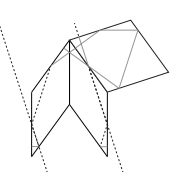

illegal
(2) 2nd extension
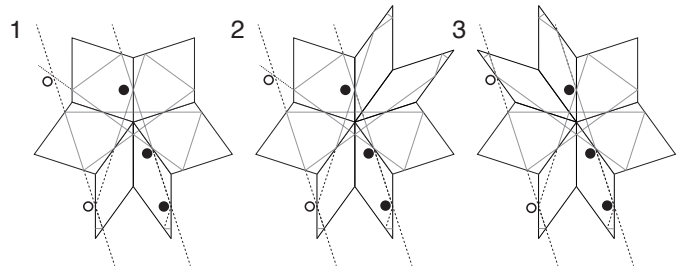

Fig. 11. Possible extensions of a tile to the opposite direction from a pair S1.

in Fig. 10(1) right. The downside of LS1 (and the symmetric version, LS2) only accepts a thin tile to form the shape S1. Thus there is no more S-gap patterns connected from S1, S2, LS1, and LS2.

Because most of the patterns forming an S-gap are S1, S2, LS1, and LS2, the other patterns appeared in an S-gap must be generated by the above process. So all possible placements except their symmetric cases along an S-gap are included.

In the same way, we consider the possible placements along an L-gap, we start from a pair L1. Although L1 contains line segments for two distinct L-gaps, they are symmetric. So it is enough to consider one pair of them. Fig. 12 (1) shows the first possible extensions. There is only one feasible placement and the next extensions of it are in Fig. 12 (2).

We consider the next step of Fig. 12 (2)-1. The only feasible placement is Fig. 12 (3)-4 and a thin tile in the next step (Fig. 12 (4)). Thus we do not need to consider the case of Fig. 12 (2)-2 separately, because the angle of the thin tile in Fig. 12 (4) is the same. The next step, only the case, Fig. 12 (5)-3, is possible and there are two options Fig. 12 (6) after the extension. L1 appears again in Fig. 12 (6)-1. The final extension in Fig. 12 (7) shows the only legal extension and the opposite side of L1 appears.

Then we consider the opposite side of L1. Fig. 13 is the possible extensions to the opposite direction from a pair L1. In Fig. 13-1, it appears the opposite 


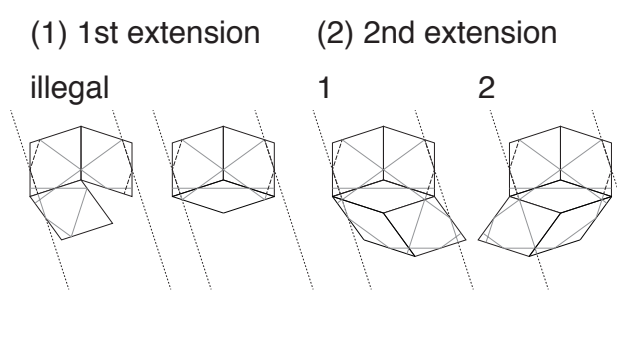

(4) 4th extension (5) 5th extension

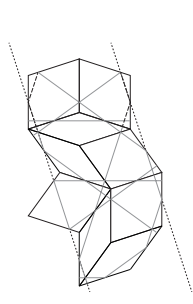

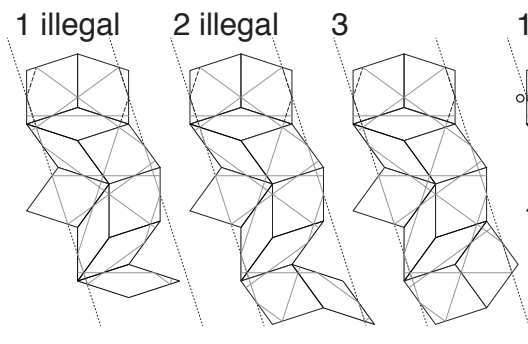

(6) 6th extension

(3) 3rd extension

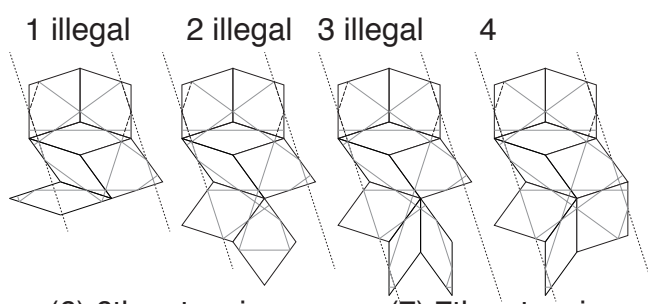

(7) 7th extension

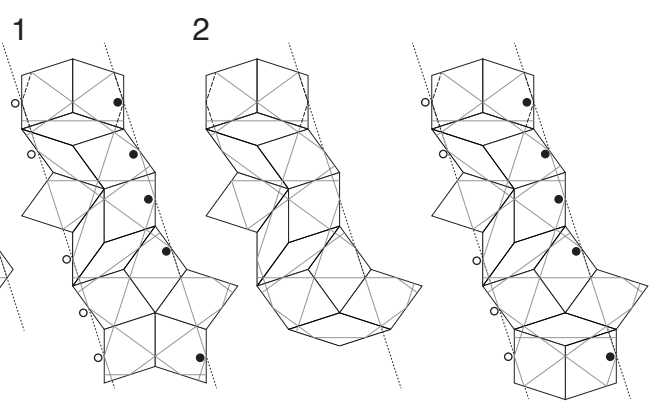

Fig. 12. The possible extensions from a pair L1.

side of L1 again and Fig. 13-2 (Fig. 13-3) has the same shape as Fig. 12 (2)-1 (Fig. 12 (2)-2), respectively. Thus all possible patterns appeared from L1 are shown in the previous figures.

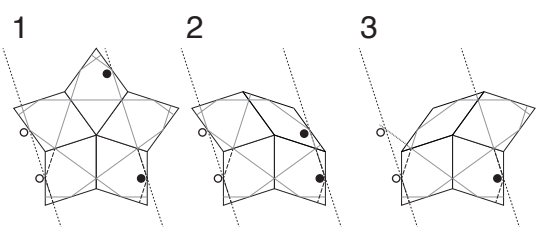

Fig. 13. Possible extension to the opposite direction from a pair L1.

The upper side of L2 (and its symmetric version) appears in Fig. 12 (2). The downside of L2 (and its symmetric version) appears in Fig. 12 (5)-3. The downside of LS1 (and the symmetric version, LS2) appears in Fig. 12 (2)-2. The upper side of LS1 (and the symmetric version, LS2) only accepts a thin tile and succeeding placements are the same as that in Fig. 12 (4). Thus there is no more L-gap patterns connected from L1, L2, L3, LS1, and LS2. 
To sum up, all possible placement of tiles along S-gaps and L-gaps are included in the above figures. Thus it is clear that the proposition agree with the all patterns in the figures.

Prop. 3 implies that a state- 1 signal coming to a side of a gap $G\left(X_{i}, X_{i+1}\right)$ takes exactly two steps to pass through the gap. Thus the following proposition holds:

Proposition 4. Let $\mathcal{A}$ be a von Neumann neighborhood growth cellular automaton on a rhombus Penrose tiling. All tiles of its initial configuration are state 0 except a filled gap of two Ammann bars $X_{i}$ and $X_{i+1}$. After two steps execution of $\mathcal{A}, G\left(X_{i+1}, X_{i+2}\right)$ and $G\left(X_{i-1}, X_{i}\right)$ are filled and all gaps outside of $X_{i+2}$ or $X_{i-1}$ are remained to be unfilled.

By Prop. 4, a sufficiently long linear wavefront of state-1 cells parallel to an Ammann bar preserve its linearity after filling the next gap. But we need to show the case of decagon. In the following, we show how each node of a filled decagon $D_{k+1}$ is formed by its previous filled decagon $D_{k}$.

Definition 4. Let $D_{i}$ be a sequence of uniaxial Ammann bar decagons. For an integer $k$, we call $D_{k}$ is filled when the state of all tiles in $D_{k}$ is 1 , except fat tiles of which longer diagonal lines are perpendicular and crossing with each line in $\partial D_{k}$. We do not concern the states of these fat tiles.

Proposition 5. Let $\mathcal{A}$ be a von Neumann neighborhood growth cellular automaton on a rhombus Penrose tiling and $D_{i}$ be a sequence of uniaxial Ammann bar decagons. Suppose all tile has state 0 in its initial configuration except a filled decagon $D_{k}$ for an integer $k$. Then after two-step executions of $\mathcal{A}, D_{k+1}$ is filled and the outside of $D_{k+1}$ is remained to be unfilled.

Proof. By Prop. 4, the outside of $D_{k+1}$ is remained to be unfilled. We need to show that all tiles in $G\left(X_{k}, X_{k+1}\right) \cap D_{k+1}$ are filled after two-step executions. It is enough to show that the closest tile (in $D_{k+1}$ ) to each vertex of $D_{k+1}$ can be reached in two-step executions from its associated vertex of $D_{k}$. Two crossing points of two pairs of two neighboring parallel Ammann bars of which angle is $4 \pi / 5$ are the candidates of vertices of $D_{k}$ and $D_{k+1}$. In the pictures of proof of Prop. 3, black circles and white circles are all such candidates of points. It is easy to check that they all have two-step distance.

Theorem 1. The edge corona limit of rhombus Penrose tilings is a regular decagon.

Proof. Let $\mathcal{A}$ be a von Neumann neighborhood growth cellular automaton on a rhombus Penrose tiling with any finite initial configuration (the number of state-1 cell is a finite natural number). We can choose a sequence of uniaxial Ammann bar decagons $D_{i}$ close to the initial configuration. Even if the given initial configuration is not connected, executing enough steps of $\mathcal{A}$, it is possible to change the shape of state- 1 cells as follows: 
1. the shape formed by the state 1 tiles is connected;

2. the shape contains a star and at least one of Ammann bar decagon $D_{i}$ for some $i$,

i.e., there exists integers $i$ and $j(>i)$ such that $D_{i}$ is filled and tiles outside of $D_{j}$ are state 0 . We denote this initial patch by $\mathcal{P}^{[0]}$. By Prop. $5, D_{n+i}$ is filled and tiles outside of $D_{n+j}$ are state 0 after $2 n$-step executions, i.e., $D_{n+i} \subset \mathcal{P}^{[2 n]} \subset$ $D_{n+j}$ holds. Because $j-i$ is a constant, $\mathcal{P}^{[n]} / n$ converges to the regular decagon $\lim _{n \rightarrow \infty} D_{[n / 2]} / n$ as $n$ tends to infinity.

\section{The difference of growth speeds}

In the previous section, we show the wavefront of a growth cellular automaton on a rhombus Penrose tilings eventually forms a regular decagon and the speed of the wavefront passing through L- or S-gap is $l / 2$ per step, $l \in\{\mathrm{S}, \mathrm{L}\}$. Although the signal propagation of the Moore neighborhood case is more fluctuating, the wavefront also forms a regular decagon and its speed is $l, l \in\{\mathrm{S}, \mathrm{L}\}$ by the existence of thin tiles along an S-gap. In the case of kite and dart tilings, it is also possible to show the similar result in the same way.

Each growth speed across a group of Ammnann bars are shown in Table 1. They actually agree with the difference of growth illustrated in Fig. 5.

Table 1. Growth speeds.

\begin{tabular}{l|l|l} 
& rhombus & kite and dart \\
\hline von Neumann & $L / 2, S / 2$ & $L / 3, S / 2$ \\
Moore & $L, S$ & $L / 2, S$
\end{tabular}

In Section 4, we pointed out that the convergence speed to a decagonal shape is very slow in the case of rhombus Moore neighborhood. To inspect the behavior, we modify the local function of the growth cellular automaton as follows:

Definition 5. A cellular automaton $\mathcal{A}_{+}$whose state set is $Q=\{0,1,2,3, \ldots\}$ $(|Q|$ is the maximum number of tiles sharing a vertex). Its local function is defined such that if the state of a focus tile is 0 then its state is changed to the sum of all non-zero cells in $\mathcal{N}$. Any tile with state $\geq 1$ never changes its state.

Fig. 14 illustrates the evolutions of $\mathcal{A}_{+}$in the case of square and rhombus Penrose tilings. A light gray (state 1) tile receives a signal from a neighboring tile and a darker tile receives two or more signals at a time from two or more neighboring tiles. Intuitively a light gray line segment shows that the existence of the fastest signal propagation at the sites. In the square cases, the line segments are the diagonal lines of a square formed by the 'light speed' of the cellular space.

In the rhombus cases, there are many light gray line segments of short length. This mainly due to the fluctuation of signal propagations caused by the existence 
of alternations of L and S gaps. But even the tiling is the same, the situation is quite different between the von Neumann and the Moore neighborhood cases. In the Moore neighborhood case, there are many self similar light gray line segments and complicated signal collisions occur. This is the reason for the slow convergence to a regular decagonal shape in the viewpoint of signal propagation of cellular automata.

As the result, employing rhombus tiling and Moore neighborhood might be more suitable for the cellular automata simulation of, for example, chemical reactions than the other cases. Because it anyway achieves the most homogeneous local signal propagation in the above options.

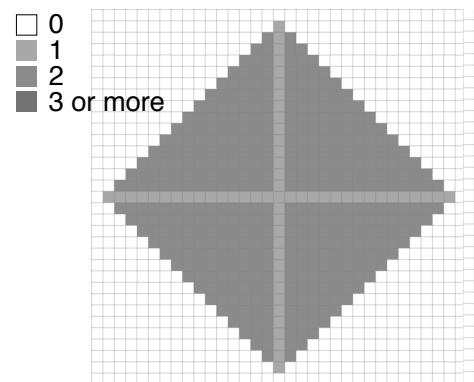

Square, Neumann

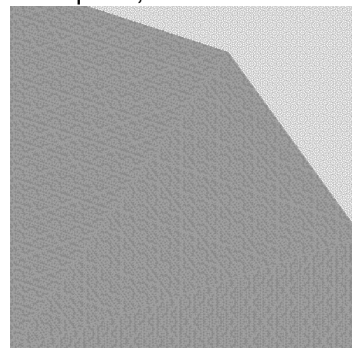

Rhombus, Neumann

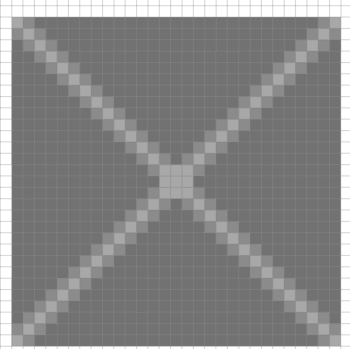

Square, Moore

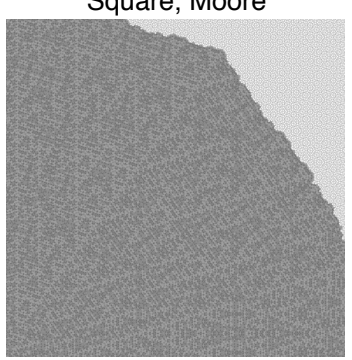

Rhombus, Moore

Fig. 14. The evolutions of $\mathcal{A}_{+}$

\section{Conclusion}

In this paper, we showed that the corona limit of Penrose tilings is a regular decagon, thanks to the existence of Ammann bars. This might suggest some relation between corona limits and windows of the cut and project scheme which generate the tilings. However we know little on this connection. This idea may fail if the cut and project scheme is non Archimedean. For the case of the chair tiling (Fig.10.1.5 in [6]), its corona limit is easily shown to be an octagon. The 
associated cut and project scheme is realized with 2-adic internal space [1] whose window is not a four dimensional hypercube.

It is an intriguing question to understand what decides the shape of the corona limit.

Acknowledgments. Shigeki Akiyama is supported by the Japanese Society for the Promotion of Science (JSPS), Grant in aid 21540012. Katsunobu Imai thanks Takahiro Hatsuda from SOLPAC Co., Ltd. for the helpful discussions and gratefully acknowledges the support of JSPS KAKENHI Grant Number 26330016 .

\section{References}

1. Baake M., Moody R.V. and Schlottmann M.: Limit-(quasi)periodic point sets as quasicrystals with p-adic internal spaces. Journal of Physics A: Mathematical and General, 31, (1988) 27.

2. Chidyagwai, P., Reiter, CA.: A local cellular model for growth on quasicrystals. Chaos, Solutions and Fractals 24 (2005) 803-812.

3. Gardner, M.: Mathematical Games - The fantastic combinations of John Conway's new solitaire game "life" (1970) 223, 120-123.

4. Gardner, M.: Extraordinary nonperiodic tiling that enriches the theory of tiles. Mathematical Games, Scientific American, January, (1977) 110-121.

5. Goucher, A. P.: Gliders in Cellular Automata on Penrose Tilings. Journal of Cellular Automata, 7 (5-6), (2012) 385-392.

6. Grünbaum, B., Shephard, C.G.: Tiling and Patterns (1987) Freeman, San Francisco.

7. Hutton, T.: Ready: a cross-platform implementation of various reaction-diffusion systems, https://github.com/GollyGang/ready.

8. Kellendonk, J., Lenz, D. and Savinien, J. (ed.), Mathematics of aperiodic order, Progress in Mathematical Physics, 309. Birkhuser/Springer, Basel, 2015. xi+428 pp.

9. Lück, R., Basic ideas of Ammann bar grids, International Journal of Modern Physics B, 7, Nos.6 \& 7 (1993) 1437-1453.

10. Mann, C., Heesch's tiling problem, Amer. Math. Monthly 111 (2004), no. 6, 509517.

11. McClure, M.: A Stohastic Cellular Automaton for Three-Coloring Penrose Tiles Computers \& Graphics 263 (2002) 519-524.

12. Owens, N., Stepney, S.: Investigation of the Game of Life cellular automata rules on Penrose tilings: lifetime, ash and oscillator statistics. Journal of Cellular Automata 53 (2010) 207-225.

13. Owens, N., Stepney, S.: The Game of Life rules on Penrose tilings: still life and oscillators. In: A. Adamatzky (Ed.) Game of Life Cellular Automata, SpringerVerlag London (2010) 331-378.

14. Schattschneider, D. and Dolbilin, N.: One corona is enough for the Euclidean Plane, In Jiř̀̀ Patera (Ed.) Quasicrystals and discrete geometry, Fields Institute Monographs, 10, AMS, Providence, RI (1998) 193-199.

15. Solomyak, B., Nonperiodicity implies unique composition for self-similar translationally finite tilings, Discrete Comput. Geom. 20 (1998), no. 2, 265-279.

16. Wolfram, S.: in A New Kind of Science (2002) Wolfram Media, 929-929. 